\title{
Global Burden of Toxocariasis: A Common Neglected Infection of Poverty
}

\author{
Sarah L. McGuinness • Karin Leder
}

Published online: 14 January 2014

(C) Springer International Publishing AG 2013

\begin{abstract}
Toxocariasis is a zoonosis of global importance, yet our understanding of the burden of human disease is limited by insufficient clinical awareness, absence of standardized diagnostic criteria and lack of coordinated epidemiological surveillance (Macpherson in Int J Parasitol 43(12-13):999-1008, 2013; Smith et al. in Trends Parasitol 25(4):182-188, 2009; Rubinsky-Elefant et al. in Ann Trop Med Parasitol 104(1):3$23,2010)$. Human infection results from zoonotic transmission of the nematode Toxocara canis, and to a lesser extent T. cati, whose definitive hosts are dogs and cats, respectively (Despommier in Clin Microbiol Rev 16(2):265-272, 2003). Toxocara canis and T. cati are distributed worldwide, and seroprevalence studies have shown that toxocariasis is especially prevalent among socioeconomically disadvantaged children in both developing and developed nations (Smith et al. in Trends Parasitol 25(4):182-188, 2009; Hotez in PLoS Negl Trop Dis 3(3):e400, 2009). Infection in humans occurs after accidental ingestion of embryonated eggs containing Toxocara larvae present in contaminated soil or food (Despommier in Clin Microbiol Rev 16(2):265-272, 2003), or by ingestion of encapsulated larvae in raw tissues of paratenic hosts, such as cows, sheep and chickens (Akao and Ohta in Parasitol Int 56(2):87-93, 2007). Clinical manifestations range from asymptomatic infection to severe organ injury, and treatment varies according to symptoms and location of the larvae (Macpherson in Int J Parasitol 43(12-13):999-1008, 2013). Improved understanding of the global health impact of toxocariasis would assist control of this common neglected infection.
\end{abstract}

S. L. McGuinness $\cdot$ K. Leder

Victorian Infectious Diseases Service, Royal Melbourne Hospital, Grattan Street, Parkville, VIC 3050, Australia

\section{K. Leder $(\bowtie)$}

Infectious Disease Epidemiology Unit, Department of Epidemiology and Preventive Medicine, Monash University, Clayton, VIC 3168, Australia

e-mail: Karin.Leder@monash.edu
Keywords Human toxocariasis $\cdot$ Toxocara canis $\cdot$ Toxocara cati $\cdot$ Visceral larva migrans $\cdot$ Ocular larva migrans .

Neurotoxocariasis $\cdot$ Covert toxocariasis

\section{Introduction}

Human toxocariasis is one of the most widespread and economically important zoonoses worldwide, yet there is a relative scarcity of public awareness of this important infection $[1 \bullet \bullet, 2 \bullet$. Toxocariasis is caused by infection with larvae of the nematode parasites Toxocara canis, and to a lesser extent $T$. cati, whose definitive hosts are dogs and cats, respectively [3]. Several other species of Toxocara may contribute to the global burden of toxocariasis, such as T. malaysiensis, found in domestic cats in Malaysia and China [4], but their importance is not well understood $[1 \bullet \cdot$. Toxocara canis and T. cati are distributed worldwide, and are particularly prevalent in the tropics, where the humid climate favours the survival of eggs in the soil [5]. In socioeconomically disadvantaged populations, poor hygiene and low rates of antihelminthic treatments to dogs increases the likelihood of human infection $[1 \bullet \bullet, 2 \bullet, 3]$. There is a range of clinical manifestations of toxocariasis in humans, but the two classical clinical syndromes described are visceral larva migrans (VLM) [6] and ocular larva migrans (OLM) [7].

\section{Life Cycle and Hosts}

\section{Definitive Hosts}

Domestic dogs are the predominant definitive host for Toxocara canis, but other canids (foxes, wolves and coyotes) can carry and transmit the infection [1••]. Domestic cats and other felids carry and transmit $T$. cati [8]. In definitive hosts, transmission can occur vertically via transplacental and transmammary 
routes, horizontally through the ingestion of embryonated eggs from the environment, and via ingestion of larvae through the consumption of paratenic hosts [9]. Rates of infection and parasite burden are highest in puppies and juvenile dogs less than 6 months of age, and in kittens [9]. After ingestion of infective $T$. canis eggs, larvae hatch in the duodenum and penetrate the intestinal walls to enter the circulatory system [10]. In pups and young dogs, larvae migrate to the pulmonary circulation where they penetrate the walls of the alveoli, migrate up the bronchial tree and are swallowed before maturing into adult worms in the small intestine. T. canis eggs first appear in dog faeces $4-5$ weeks after infection [11] and embryonate in the environment within $2-3$ weeks if the humidity and temperature are suitable [2•]. Toxocara eggs can tolerate and develop at a wide range of ambient temperatures [5]. In older animals, larvae typically migrate to other tissues, where they remain developmentally arrested in an encysted form that can persist for long periods [2•]. Reactivation of encysted larvae in late pregnancy leads to infection in puppies via the transplacental and transmammary routes [9]. Larvae ingested through the consumption of paratenic hosts in most cases develop directly into adult worms in the intestinal tract [9].

\section{Paratenic Hosts}

Other animal species are susceptible to infection through ingestion of embryonated eggs and can act as paratenic hosts for Toxocara species. Recognized paratenic hosts include chicken, cattle, pigs, rodents and birds [10]. While larvae cannot mature to adults in these hosts, they can migrate to tissues and persist in a developmentally arrested form, which can remain viable for up to 10 years [10]. Human infection may occur after consumption of raw meat from infected paratenic hosts, and ingestion of paratenic hosts also plays an important role in $T$. cati infection in adult cats [1••].

\section{Human Infection}

Infection in humans occurs after accidental ingestion of embryonated eggs containing Toxocara larvae present in contaminated soil or food [3], or by the ingestion of encapsulated larvae in the raw tissues of paratenic hosts, such as cows, sheep and chickens [12]. Children are more frequently infected than adults as they are more often exposed to Toxocara eggs, in settings such as sandboxes and playgrounds contaminated with dog and cat faeces [3]. The life cycle of Toxocara is outlined in Fig. 1. Embryonated eggs from the environment or raw meat from paratenic hosts are ingested and larvae hatch in the small intestine. Larvae penetrate the intestine, enter the circulation and are able to migrate to a variety of tissues, where they can activate host inflammatory responses leading to mechanical and immunopathological damage to tissues, and a heterogeneous range of clinical manifestations [13].

\section{Epidemiology}

\section{Global Seroprevalence}

Seroprevalence data have been collected from a range of settings, and prevalence estimates vary widely depending on the population demographics, age of the subjects and geographical location $[2 \bullet$. In industrialized environments, relatively low seroprevalance rates have been reported, for example $0.7 \%$ in New Zealand, $1.6 \%$ in Japan and $2.4 \%$ in Denmark $[2 \cdot 12,14]$. In contrast, much higher seroprevalence rates have been reported in tropical, less industrialized settings, for example $92.8 \%$ in La Reunion, $81 \%$ in Nepal and $63.2 \%$ in Indonesia $[2 \cdot 13]$. Interestingly, seroprevalence in the USA has been reported to be as high as $13.9 \%$ [15], suggesting that toxocariasis is a significant public health problem even in highly industrialized settings [16]. Valid comparison of data between countries is limited by a number of factors, including the lack of standardization of clinical disease definitions, variation in detection methods, and demographic diversity of populations studied [13, 17].

\section{Risk Factors}

There is considerable variability in reported risk factors for toxocariasis. Factors identified as contributing to heavily contaminated environments include poor sanitation and hygiene, lack of education, and low socioeconomic status [2•]. Young age is consistently reported to be associated with a greater risk of toxocariasis, and is most commonly attributed to increased environmental exposure and behavioural factors [15]. Having a dog as a pet is recognized as a risk factor in a number of studies [18•]. However, infection is often acquired in settings outside the home (such as in public parks and playgrounds) and is not dependent on dog ownership [2•, 19]. Consumption of raw meat has been linked to an increased risk of toxocariasis [10], but its epidemiological importance as a risk factor has yet to be clarified [1••]. The incidence of Toxocara infection in travellers is low [20] and travel to countries with high seroprevalance does not appear to be a risk factor for toxocariasis.

\section{Immunopathogenesis}

While Toxocara larvae are unable to moult, grow or replicate in the human host, parasites can remain viable for at least 7 years after infection [17]. During this time they can migrate through tissues, causing mechanical and immunopathological damage [3].

The extraordinary survival capacity of Toxocara larvae has been attributed to two molecular strategies evolved by the parasite $[21 \bullet]$. The first is the release of 'excretory-secretory' 
Fig. 1 Life cycle of Toxocara canis and Toxocara cati, the causal agents of toxocariasis (image courtesy of CDC-DPDx)

\section{Toxocariasis}

(Toxocara canis, Toxocara cati)

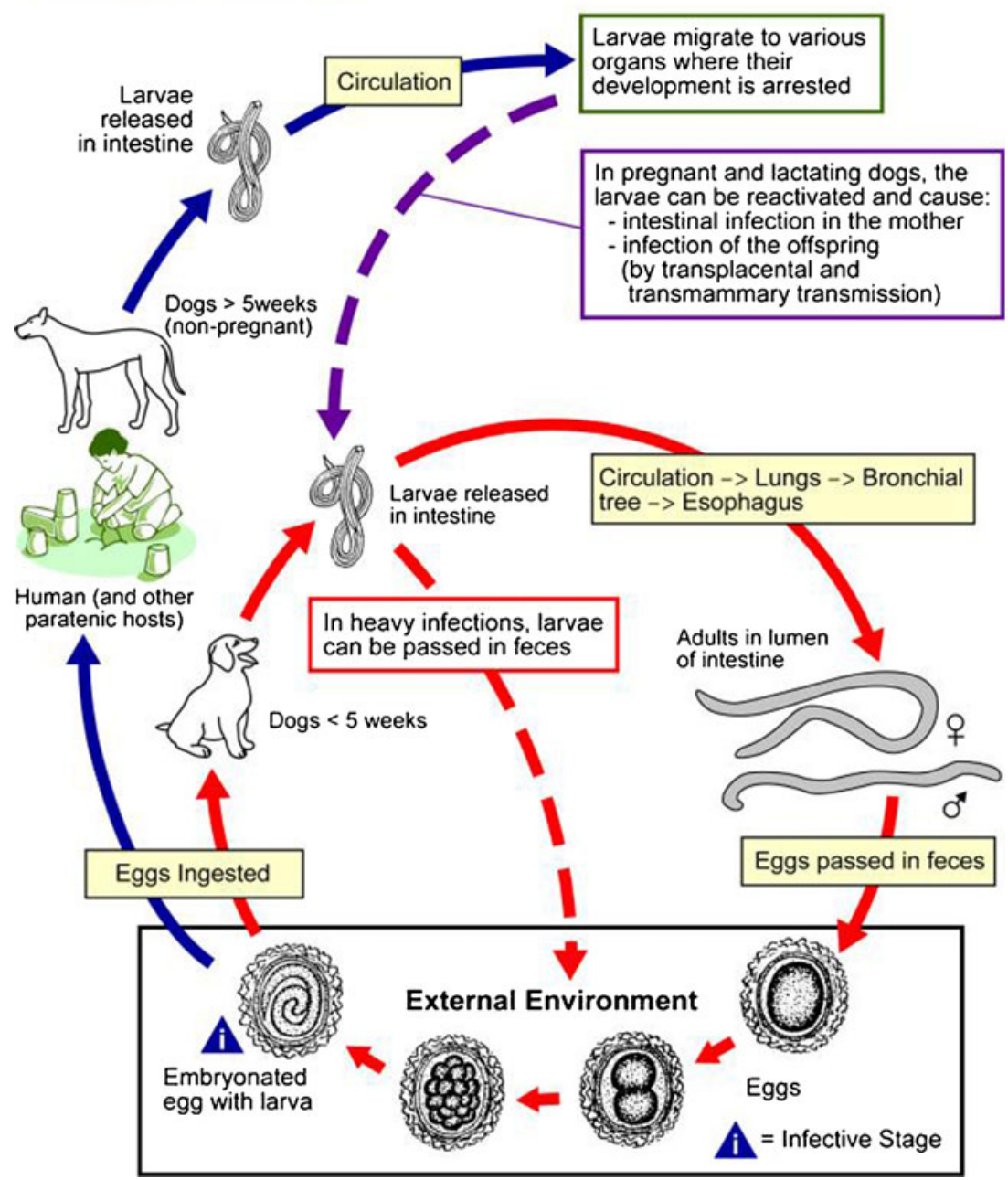

products including lectins, mucins and enzymes that interact with and modulate host immunity, such as by interfering with leucocyte extravasation into affected sites [21•]. The second is the production of a loosely attached mucin-rich surface coat to which host antibodies and cells adhere, which the parasite can detach, leaving behind an inflammatory reaction around a newly vacated focus $[21 \bullet]$.

The human immune response in toxocariasis is characterized by a T-helper type-2 (Th2) response to the parasite's 'excretory-secretory' antigens, resulting in marked eosinophilia, production of cytokines including IL-4, IL-5 and IL13 , and $\mathrm{IgG} / \operatorname{IgE}$ hypergammaglobulinaemia $[13,21 \bullet]$.

The clinical manifestations of larval migration vary widely depending on the number of larvae ingested, the age of the host, the location of the migrating larvae, and other hostparasite interaction factors which are only partially understood $[3,13,22]$. The liver, lungs, and central nervous system, including the eyes, appear to be the organs most sensitive to invasion. In self-limited infections, the larvae become encapsulated in the musculature and liver, and symptoms subside $[1 \bullet \bullet$. Migration of larvae through tissues can lead to eosinophilic infiltration, granuloma formation, or eosinophilic abscess [22]. The characteristic pathological finding in OLM and VLM is eosinophilic granulomas with central necrosis surrounded by a mixed inflammatory infiltrate with numerous eosinophils [3, 23]. Granulomatous lesions are often multiple, and recognizable larvae are uncommon [23].

\section{Clinical Manifestations}

There is a varied clinical spectrum of toxocariasis in humans, ranging from asymptomatic infection to severe organ injury, with symptoms related to the migration of third-stage larvae of Toxocara spp. via the bloodstream to the internal organs. In general, most infections are self-limiting as larvae become encapsulated in the musculature and liver $[1 \bullet \bullet]$. 
Although toxocariasis is rarely fatal, two severe clinical syndromes are classically recognized: VLM (systemic disease caused by larval migration through major organs) and OLM (in which the pathological effects on the host are restricted to the eye and the optic nerve). Over the last few decades, it has become recognized that the spectrum of clinical disease is more diverse. Two less severe syndromes have been described in humans: 'covert toxocariasis', seen mainly in children, and 'common toxocariasis', seen predominantly in adults [2•, 24-26]. Another form of infection is neurotoxocariasis [27, 28]

A slightly different way to classify human toxocariasis into four major forms has recently been proposed: asymptomatic, covert, systemic (VLM, classic or incomplete), and compartmentalized (ocular and neurological toxocariasis) [29]. Both classifications recognize that in each individual, the manifestations are determined by a number of factors including the parasite load, sites of larval migration, duration of migration, and the host's inflammatory response [2•]. Except for mechanical injury caused by migrating larvae, such as in the eye or brain, an immunopathogenic mechanism underlies most clinical manifestations of toxocariasis [29].

\section{Covert or Common Toxocariasis}

Many individuals tested in population-based surveys and found to be positive for Toxocara antibodies on serological testing are completely asymptomatic or have only mild and nonspecific symptoms. A case-control study performed in the 1980s in children resulted in the description of a clinical entity named 'covert toxocariasis' in which various combinations of nonspecific symptoms and signs were seen, including fever, abdominal pain, anorexia, nausea, vomiting, hepatomegaly, cough, headache, lethargy, behavioural and/or sleep disturbances, skin symptoms, limb pains, and lymphadenitis [25, 30]. This presentation was generally associated with quite high titres of anti-Toxocara antibodies, with or without eosinophilia. Another study in adults led to the definition of 'common toxocariasis', referring to a syndrome comprising chronic weakness, shortness of breath, abdominal pain, rash, itch, urticaria and arthralgia, often with eosinophilia, high IgE levels, and high titres of Toxocara-specific antibodies [26, 31]. These two syndromes may represent age-dependent variations of the same clinical entity, and the nonspecific symptoms are likely to be due to continued stimulation of the host immune system by parasite antigens [17, 29, 32].

\section{Visceral Larva Migrans}

Classical VLM typically occurs in children aged $2-7$ years, who often have a history of geophagia or exposure to dogs at home, but it also occurs in adults [12]. Classical VLM is a severe systemic form associated with a high-intensity infection or repeated exposure to larvae. Immediate hypersensitivity response to the death of larvae is thought to underlie most of the symptoms [3]. While classic full-blown, severe VLM is relatively uncommon, an incomplete VLM syndrome with lower severity and with only some signs of the classic VLM form occurs much more frequently.

Clinical signs are mainly hepatic and pulmonary, classically resulting in fever, cough, wheeze, shortness of breath, moderate hepatomegaly, mild splenomegaly, abdominal pain and anorexia [18•]. Chronic cough is a relatively common finding in infected children. The liver is the organ most commonly affected, with typical hepatic granulomas showing multinucleated giant cells and epithelioid cells surrounding necrotic debris or amorphous eosinophilic material [2•]. Neurological and cutaneous manifestations, discussed further below, may also be seen in VLM. Generalized lymphadenopathy may also be present. Eosinophil counts can be very high, not uncommonly exceeding $50 \%$, which, if prolonged, can lead to complications such as pulmonary fibrosis and eosinophilic myocardial fibrosis. Other less common manifestations include nephrotic syndrome, arthritis and myositis $[2 \cdot 33]$.

\section{Ocular Larva Migrans}

The clinical condition now known as OLM was first described by Wilder in 1950 who found nematode larvae during the histological analysis of 24 eyes that had been enucleated from children with suspected retinoblastoma [7]. These were subsequently identified as $T$. canis larvae.

OLM is much less common than VLM. Ocular involvement often occurs as the sole manifestation of toxocariasis, and is thought to be more likely to occur in association with low intensity of infection. The underlying hypothesis is that OLM occurs when there are insufficient antigenic stimuli for a protective immune response, which allows unlimited migration of the Toxocara larvae [29]. However, systemic and ocular manifestations of toxocariasis have occasionally been reported in the same patient, possibly when there are very high doses of invasive larvae that overwhelm the liver's ability to serve as a larval filter $[34,35]$.

OLM is characterized by an eosinophilic immune response to migration of third-stage larvae into the eye. The clinical presentation of OLM depends on the exact anatomical site involved and the associated immune response elicited in the host. Males tend to be affected more frequently than females, and children are affected more commonly than adults [36]. However, compared with VLM, OLM tends to affect older children and adolescents, with a mean age at onset of 7.5 years [36].

Clinical manifestations are usually unilateral. Common presentations are with progressively deteriorating vision over days to weeks, strabismus and leucocoria. The clinical signs seen most often and the major cause of vision loss are vitreous inflammation, macular oedema, and formation of fibrous 
traction bands that lead to retinal detachment [37]. Unilateral ocular inflammatory peripheral or posterior retinitis, retinal, macula or optic nerve granuloma, or circumscribed endophthalmitis may be found. Retinal granuloma can be mistaken for a retinoblastoma. Other clinical presentations include papillitis, uveitis, keratitis, or a motile intraocular nematode. Opacification of the vitreous humour can be accompanied by deposits of immune complexes, often in the periphery ("snowballs") [38••]. It has been suggested that the clinical presentation of OLM may be age-dependent, with diffuse toxocaral endophthalmitis occurring most frequently in patients between 2 and 9 years of age, retinal granuloma between 6 and 14 years of age and pars planitis between 6 and 40 years of age [39]. Importantly, migration of even a single larva into the eye can lead to detachment of the macula, which can cause irreversible blindness. Secondary glaucoma can also develop.

Neurological Manifestations

Neurotoxocariasis arises from the invasion by Toxocara larvae of the brain and spinal cord. In the brain, Toxocara larvae are not encapsulated and their migratory tracks result in small areas of necrosis and some inflammatory infiltration, with secondary granuloma formation $[29,40,41]$. Cerebral lesions are predominantly located in the white matter [10], and can result in meningitis, encephalitis, cerebritis or cerebral vasculitis. Clinically, patients present with a range of symptoms including headache, photophobia, weakness, ataxia, paresis, visual impairment, confusion and seizures [27, 28].

Toxocariasis has also been implicated as a cause of epilepsy, with a recently published meta-analysis showing a significant association between epilepsy and Toxocara seropositivity [42], and almost a doubling of seizures in those with Toxocara antibodies. However, further research to confirm causality is needed. Some studies have also linked T. canis seropositivity with impaired cognitive development and neuropsychological disturbances [19, 43], although confirmation of an association again requires more research [2•].

Manifestations of the toxocaral involvement of the peripheral nervous system include cranial nerve palsy and radiculitis $[27,28]$.

\section{Cutaneous Manifestations}

Cutaneous symptoms are relatively frequent in patients with Toxocara infection, especially those with VLM and common toxocariasis [44]. While sometimes dermatological symptoms occur together with other clinical findings related to infection and/or with eosinophilia, they can also be the only indication of toxocariasis. Chronic urticaria is the most common skin problem described. Other cutaneous manifestations include chronic pruritus, chronic prurigo, transient rash, different forms of eczema, hypodermic nodules and vasculitis [44].
Association with Asthma

Although VLM is often associated with wheezing, it is unclear if Toxocara infection predisposes to or induces asthma, with both positive associations and negative epidemiological studies reported [2•]. Asthma-like symptoms can result from larval migration through the lungs, but a role has also been proposed for parasite-induced atopy $[45,46]$. Experimental T. canis infection can exacerbate allergic airway inflammation in mice, possibly by enhancing Th2-type immune responses to aeroallergens [47]. A recent study in Cuba suggested that Toxocara seropositivity is associated with asthma, but appears to nullify the apparent association between atopy and asthma [48].

\section{Diagnosis}

Diagnosis of toxocariasis involves clinical, laboratory, radiological, pathological and serological aspects. Five major markers of symptomatic toxocariasis have been suggested: (1) the characteristics and history of the patient; (2) clinical symptoms and signs, (3) positive serology, (4) eosinophilia, and (5) increased levels of the IgE class of immunoglobulins [29].

\section{Biopsy for Histopathology and Molecular Testing}

Histopathological examination of biopsy specimens to look for Toxocara larvae by microscopy is the only way to establish a definitive diagnosis, but tissue sampling is invasive so is not commonly performed. Additionally, it is difficult to know exactly where to biopsy to obtain a specimen containing larvae. Should a larva be present, it is identified by its squat appearance, length of approximately $400 \mu \mathrm{m}$ and width of 18 $20 \mu \mathrm{m}[38 \bullet \bullet]$. Even if a larvae or part of a larva is successfully recovered, it is often impossible to distinguish between Toxocara larvae and those of other ascaridoid species [49•]. While not often diagnostic, biopsies often will show eosinophilic inflammation which supports the diagnosis.

In a research context, molecular diagnostic methods using PCR-based techniques can be performed on biopsy specimens and can help with species differentiation. However, the small inocula in most human infections means that the probability of obtaining a tissue sample containing any larval antigenic tissue is very low [38••]. Because of this low probability, the invasiveness of specimen collection and its limited availability, the PCR approach is not currently used in clinical practice [38••].

\section{Eosinophilia}

Toxocara infection has been reported to be the main cause of unknown peripheral blood eosinophilia, usually with eosinophil counts over 500 cells $/ \mathrm{mm}^{3}$ or $>10 \%$ of the white blood 
cell count [50]. In the classic VLM syndrome, eosinophilia is usually present (in $>90 \%$ of patients), with levels commonly exceeding 10,000 cells $/ \mathrm{mm}^{3}$ and sometimes exceeding $70 \%$ of the total white blood cell count. However, in common or covert toxocariasis, eosinophilia may be absent, and typical levels are lower (approximately $400-1,500 \mathrm{cells} / \mathrm{mm}^{3}$ ) [26, $38 \bullet \bullet$. The intensity of eosinophilia may therefore have some diagnostic value, with a count above 3,000 cells $/ \mathrm{mm}^{3}$ being typical of classic VLM [29]. In over $80 \%$ of patients with ocular toxocariasis, there is no peripheral blood eosinophilia, presumably because of the low larval load [29]. Eosinophilia may also be absent in patients with long-standing infections, and in those with only chronic skin symptoms.

\section{Other Laboratory Markers}

About $50 \%$ of patients have hypergammaglobulinaemia of immunoglobulin M (IgM), IgG, and IgE classes. Other findings, especially in VLM, include leucocytosis, anaemia, hypoalbuminaemia, and increased isohaemagglutinin titres to the A and B blood-group antigens. Eosinophilic granulomatous hepatitis may develop leading to abnormalities in liver function tests, including elevated transaminases and/or alkaline phosphatase. Pulmonary involvement may result in eosinophilia in bronchoalveolar lavage fluid. With central nervous system involvement, the cerebrospinal fluid may show eosinophils. Of note, the presence of ova and parasites in the stool is not useful for diagnosis because Toxocara does not replicate in the human gut.

\section{Serological Diagnosis}

In experimentally infected animals, seroconversion has been detected between 4 days and 4 weeks after infection [10]. Early enzyme-linked immunosorbent assays (ELISAs) used extracts of $T$. canis embryonated eggs as antigen. Subsequent improvements in methods for maintaining $T$. canis larvae in culture enabled development of an ELISA for detection of IgG antibodies directed against the larval excretory-secretory antigens of $T$. canis (TES antigens). The IgG TES ELISA is now the standard test used for toxocariasis immunodiagnosis $[38 \bullet \bullet$.

There are several commercial IgG TES ELISA kits available, but the variable quality of the antigens used in the ELISA kits and the variability in the ELISA titres used as cut-off for positivity complicate both interpretation of seroprevalence data and confirmation of the diagnosis in an individual. Overall, for VLM and some forms of covert toxocariasis, the excretory-secretory antigen enzyme immunoassay is said to have a sensitivity of about $80 \%$ at a titre greater than $1: 32$, and a specificity of $90-95 \%$, but the sensitivity is lower for OLM and in patients with chronic cutaneous manifestations of toxocariasis $[3,16,29,44,51]$.
Blood samples which test positive by TES ELISA must be confirmed by western blotting (WB) [38••]. WB is based on the use of fractionated, native, TES antigens. Reactivity to bands of low molecular weight $(24-32 \mathrm{kDa})$ has been found to be specific for $T$. canis infection $[1 \bullet, 17,13,52]$. WB has both better sensitivity and increased specificity compared to ELISA [38••], but it is also more expensive and labourintensive than ELISA.

A problem with serological testing is that false-positive results are common, especially in areas where other parasite infections are prevalent which crossreact in tests for Toxocara spp. Furthermore, antigenic crossreactivity between $T$. canis and $T$. cati larvae means that serological methods are unable to discriminate between these two infections. Another important limitation of serology is that it cannot distinguish between antibodies from a current active infection and those that remain from a resolved infection. It is also not possible to assess the likely timing of infection using levels of specific IgM because IgM antibodies are found throughout the course of infection [38••].

\section{Other Tests}

Eosinophil cationic protein (ECP) and other granular cationic proteins are released when eosinophils are activated. The levels of ECP have been used to assess the presence of a current active toxocaral infection, even in patients with no blood eosinophilia [53], and may be useful particularly in monitoring response to treatment.

Testing for specific IgE antibodies and for IgG subclass antibodies to glycan of TES antigens have also been examined. IgE tests may have slightly greater specificity for toxocariasis, but overall do not seem to greatly enhance diagnosis $[29,38 \bullet \cdot$. Regarding IgG subclasses, specific IgG2 gives the greatest sensitivity, whereas IgG4 has the best specificity $[13,54,55]$. Detection of circulating TES antigens is another method that has been tried using a sandwich ELISA, but because most patients have a very low larval load and also because larvae can be entrapped and destroyed inside granulomas, both sensitivity and specificity are poor [38・•, 56]. Recombinant antigen tests have been assessed using an ELISA for specific IgG. A sensitivity of $92.3 \%$ and specificity of $89.6 \%$ has been reported using a $30-\mathrm{kDa}$ antigen [57]. However, further research is needed before single DOT ELISAs can be widely used. Quantification of the avidity of specific IgG may also be useful, but further studies are again needed $[38 \bullet \bullet$.

\section{Diagnosis of OLM}

The diagnosis of OLM is usually suggested by relevant clinical findings. Serological confirmation should be attempted, but as mentioned above, antibodies are often undetectable, 
possibly due to the low parasite load $[58,59]$. Using a lower cut-off point for serological testing increases the sensitivity, but the best way to improve diagnosis is to look for specific antibodies via TES ELISA or WB in the aqueous humour [60]. Intraocular production of antibodies to Toxocara can be assessed by comparing serum and aqueous humour samples obtained simultaneously. If the results are discordant, with serum negative but aqueous humour positive for antibodies, this indicates intraocular synthesis of specific anti-ES antigen $\operatorname{IgG}[38 \bullet \bullet, 61]$. The Goldmann-Witmer (GW) coefficient can then be calculated as: (level of specific $\operatorname{IgG}$ in aqueous humour/level of specific IgG in serum)/(total IgG in aqueous humour/total IgG in serum). Intraocular production of specific antibodies is defined by a GW coefficient $>3.0$ [62] .

Similarly, for neurological toxocariasis, cerebrospinal fluid and serum should be tested simultaneously, and both specimens should be examined for Toxocara antibodies and eosinophils.

\section{Imaging Techniques}

Imaging techniques are useful in assisting with the diagnosis of VLM and OLM. On CT or MR imaging, hepatic lesions usually appear as multiple, ill-defined, oval lesions measuring $1.0-1.5 \mathrm{~cm}$ in diameter, seen best on the portal venous phase in dynamic contrast-enhanced CT and MR images [63]. An enhancing rim or enhancing nodules are sometimes observed. On ultrasonography, these lesions appear as oval hypoechoic lesions which can be differentiated from metastases as they tend to be more uniform in size, have a nonspherical shape and fuzzy margins [63]. However, toxocariasis and other parasitic infections in the liver such as Bayliascaris procyonis, Capillaria hepatica, Ascaris species and some Ancylostoma species cannot be differentiated on the basis of the imaging findings [63]. On follow-up imaging, the lesions may have improved or may sometimes have moved, reflecting migration of larva in the liver. Eosinophilic cholecystitis and ascites are also occasionally seen [2•]. Pulmonary lesions appear on CT images as multifocal subpleural nodules with halos or groundglass opacities and ill-defined margins. Transient pulmonary infiltrates are also characteristic [12].

Imaging techniques can also assist with the diagnosis and treatment of OLM. Optical coherence tomography, fluorescein angiography, CT and ocular ultrasonography using transducers can be used to indicate the location of the larva. Ocular ultrasonography may show a vitreous band or a membrane extending between the posterior pole and a highly reflective peripheral mass, and may help differentiate OLM from other eye diseases $[1 \bullet \bullet, 64]$.

$\mathrm{CT}$ and MRI findings in neurotoxocariasis usually show diffuse cortical, subcortical or white matter lesions or multifocal, circumscribed, contrast-enhancing hyperintense areas on T2-weighted images [27, 28, 41]. Associated focal meningeal enhancement adjacent to the lesion may also be seen. With spinal cord involvement, solitary or multiple hyperintense lesions on T2-weighted images, which strongly enhance on T1-weighted images after administration of contrast agent have been reported in the posterior segment of the cord [41].

\section{Treatment}

Treatment recommendations in humans vary according to symptoms and location of the larvae. It is generally suggested that symptomatic patients with VLM, OLM and neurotoxocariasis be given specific anthelmintic treatment. Patients with cutaneous manifestations associated with positive serology for toxocariasis are usually also treated. However, there are few controlled trials of anthelmintic drugs for toxocariasis, and since parasitological cure cannot be assessed, the end-point used in published trials has been a decrease in the severity of the clinical signs and symptoms [2•]. While treatment may not be needed if there are minimal symptoms, it can also be argued that anthelmintic therapy should be considered regardless of symptoms in patients in whom eosinophilia and serology are at least moderately positive because of the risk of larvae localizing in the brain during the course of an infection [29].

Albendazole is the most commonly used drug for treatment. The recommended dose is $400 \mathrm{mg}$ orally twice daily for 5 days $(10-15 \mathrm{mg} / \mathrm{kg}$ daily in two divided doses for 5 days). Albendazole is preferred over other drugs such as thiabendazole and diethylcarbamazine because of better tolerability. The main second-line alternative is mebendazole, given at a dose of $100-200 \mathrm{mg}$ orally twice daily for 5 days. Both of these agents should be taken with a fatty meal to improve absorption, and both have resulted in reductions in clinical symptoms of approximately $50 \%[10,65]$. Of note, ivermectin, has not shown significant efficacy in the treatment of toxocariasis and is not recommended.

Treatment may have to be repeated, depending on the biological and clinical response. Although the use of an anthelmintic reduces the number of Toxocara larvae, it may have less or no immediate effect on allergic reactions related to toxocariasis, and allergic or atopic conditions may actually worsen during or shortly after treatment due to release of antigen [29]. To reduce inflammation and control hypersensitivity reactions to dying larvae, corticosteroids are frequently added, particularly for the treatment of ocular and neurological manifestations. In patients with chronic skin manifestations, an antihistamine such as cetirizine may be used [44].

In treatment of OLM, sight-threatening ocular inflammation as the result of OLM requires aggressive anti-inflammatory therapy with corticosteroids (e.g. prednisone 
$0.5 \mathrm{mg} / \mathrm{kg} / \mathrm{day})$, combined with prolonged albendazole treatment $(800 \mathrm{mg} /$ day in adults and $400 \mathrm{mg} /$ day in children) for 2-4 weeks [66]. Surgical treatment may be required for retinal detachment or intravitreal fibrovascular membrane proliferation $[2 \cdot]$.

\section{Follow-up After Treatment}

For individuals who present with eosinophilia, the most suitable method for follow-up of clinical toxocariasis is the eosinophil count, which decreases within 1 month of treatment [67]. Follow-up after treatment is not possible using detection of specific IgG, because the kinetics of this class of immunoglobulin are very slow, even after successful treatment [68]. On average, the TES ELISA is thought to remain positive for 2.7 years and the WB for over 5 years $[38 \bullet, 67]$. Some studies have suggested that significant decreases in $\mathrm{IgE}$ antibody plus eosinophil counts during the first year after treatment are helpful parameters for patient follow-up after therapy [69]. However, a recent review of Toxocara diagnosis concluded that it is not possible to assess the age or activity of Toxocara infection using specific IgG, specific IgM, IgE, IgA, IgG4 or circulating antigen levels [38••]. Thus the diagnosis of active versus inactive toxocariasis relies largely upon indirect findings such as the presence of otherwise unexplained symptoms and eosinophilia $[1 \bullet \bullet]$. The only other test that is thought to demonstrate some utility for distinguishing between active and past toxocaral disease is assessment of the level of ECP [70].

\section{Conclusion}

Globally, toxocariasis remains one of the most widespread and economically important zoonoses to affect humans. The parasite has an extraordinary capacity to survive for long periods, both in the environment and in the host, and has adapted to numerous domestic and wild definitive and paratenic host species. The ubiquitous distribution of definitive hosts (dogs and cats) living in close proximity to humans results in a worldwide distribution of disease. Epidemiological studies suggest that the greatest burden of disease is found in socioeconomically disadvantaged children, with links to poor sanitation and hygiene and lack of education. Clinical manifestations of toxocariasis vary widely and diagnosis is complex, involving clinical, laboratory, radiological, pathological and serological aspects, many of which are not available to those most at risk of infection. Increasing the global awareness of toxocariasis will be an important step in efforts to combat this disease. Greater understanding of the epidemiology and global burden of toxocariasis is needed to allow the development and implementation of programmes to treat and control this neglected infection of poverty.

\section{Compliance with Ethics Guidelines}

Conflict of Interest Sarah L. McGuinness declares that she has no conflict of interest.

Karin Leder has received travel/accommodation expenses covered or reimbursed by GSAK and SanofiPasteur.

Human and Animal Rights and Informed Consent This article does not contain any studies with human or animal subjects performed by any of the authors.

\section{References}

Papers of particular interest, published recently, have been highlighted as:

- Of importance

•- Of major importance

1.• Macpherson CNL. The epidemiology and public health importance of toxocariasis: A zoonosis of global importance. Int J Parasitol. 2013;43(12-13):999-1008. Review of the transmission, epidemiology, diagnosis and clinical syndromes of toxocariasis.

2. Rubinsky-Elefant G, Hirata CE, Yamamoto JH, Ferreira MU. Human toxocariasis: diagnosis, worldwide seroprevalences and clinical expression of the systemic and ocular forms. Ann Trop Med Parasitol. 2010;104(1):3-23. An excellent review of the epidemiology, clinical spectrum, and current laboratory diagnosis of human toxocariasis.

3. Despommier D. Toxocariasis: clinical aspects, epidemiology, medical ecology, and molecular aspects. Clin Microbiol Rev. 2003;16(2):265-72.

4. Gibbons LM, Jacobs DE, Sani RA. Toxocara malaysiensis n. sp. (Nematoda: Ascaridoidea) from the domestic cat (Felis catus Linnaeus, 1758). J Parasitol. 2001;87(3):660-5.

5. Azam D, Ukpai OM, Said A, Abd-Allah GA, Morgan ER. Temperature and the development and survival of infective Toxocara canis larvae. Parasitol Res. 2012;110(2):649-56.

6. Beaver PC, Snyder CH, Carrera GM, Dent JH, Lafferty JW. Chronic eosinophilia due to visceral larva migrans; report of three cases. Pediatrics. 1952;9(1):7-19.

7. Wilder HC. Nematode endophthalmitis. Trans Am Acad Ophthalmol Otolaryngol. 1950;55:99-109.

8. Fisher M. Toxocara cati: an underestimated zoonotic agent. Trends Parasitol. 2003;19(4):167-70.

9. Overgaauw PA, van Knapen F. Veterinary and public health aspects of Toxocara spp. Vet Parasitol. 2013;193(4):398-403.

10. Strube C, Heuer L, Janecek E. Toxocara spp. infections in paratenic hosts. Vet Parasitol. 2013;193(4):375-89.

11. Schnieder T, Laabs EM, Welz C. Larval development of Toxocara canis in dogs. Vet Parasitol. 2011;175(3-4):193-206.

12. Akao N, Ohta N. Toxocariasis in Japan. Parasitol Int. 2007;56(2): 87-93.

13. Fan C-K, Liao C-W, Cheng Y-C. Factors affecting disease manifestation of toxocarosis in humans: genetics and environment. Vet Parasitol. 2013;193(4):342-52.

14. Zarkovic A, McMurray C, Deva N, Ghosh S, Whitley D, Guest S. Seropositivity rates for Bartonella henselae, Toxocara canis and Toxoplasma gondii in New Zealand blood donors. Clin Exp Ophthalmol. 2007;35(2):131-4.

15. Won KY, Kruszon-Moran D, Schantz PM, Jones JL. National seroprevalence and risk factors for zoonotic Toxocara spp. infection. Am J Trop Med Hyg. 2008;79(4):552-7. 
16. Hotez PJ, Wilkins PP. Toxocariasis: America's most common neglected infection of poverty and a helminthiasis of global importance? PLoS Negl Trop Dis. 2009;3(3):e400.

17. Smith H, Holland C, Taylor M, Magnaval JF, Schantz P, Maizels R. How common is human toxocariasis? Towards standardizing our knowledge. Trends Parasitol. 2009;25(4):182-8.

18. Carvalho EA, Rocha RL. Toxocariasis: visceral larva migrans in children. J Pediatr (Rio J). 2011;87(2):100-10. Recent review of the risk factors, symptoms, laboratory and imaging tests useful for establishing the diagnosis of VLM in children. It also highlights the importance of diagnosis and treatment in order to prevent complications in the eyes, liver and other organs.

19. Jarosz W, Mizgajska-Wiktor H, Kirwan P, Konarski J, Rychlicki W, Wawrzyniak G. Developmental age, physical fitness and Toxocara seroprevalence amongst lower-secondary students living in rural areas contaminated with Toxocara eggs. Parasitology. 2010;137(1): 53-63.

20. Baaten GG, Sonder GJ, van Gool T, Kint JA, van den Hoek A. Travel-related schistosomiasis, strongyloidiasis, filariasis, and toxocariasis: the risk of infection and the diagnostic relevance of blood eosinophilia. BMC Infect Dis. 2011;11:84.

21. Maizels RM. Toxocara canis: molecular basis of immune recognition and evasion. Vet Parasitol. 2013;193(4):365-74. Summary of current knowledge around the molecular basis of immune recognition of Toxocara spp and their ability to evade the immune system.

22. Beaver PC. The nature of visceral larva migrans. J Parasitol. 1969;55(1):3-12.

23. Kaplan KJ, Goodman ZD, Ishak KG. Eosinophilic granuloma of the liver: a characteristic lesion with relationship to visceral larva migrans. Am J Surg Pathol. 2001;25(10):1316-21.

24. Bass JL, Mehta KA, Glickman LT, Eppes BM. Clinically inapparent Toxocara infection in children. N Engl J Med. 1983;308(12): $723-4$.

25. Taylor MR, Keane CT, O'Connor P, Mulvihill E, Holland C. The expanded spectrum of toxocaral disease. Lancet. 1988;1(8587): 692-5.

26. Glickman LT, Magnaval JF, Domanski LM, Shofer FS, Lauria SS, Gottstein B, et al. Visceral larva migrans in French adults: a new disease syndrome? Am J Epidemiol. 1987;125(6):1019-34.

27. Finsterer J, Auer H. Neurotoxocarosis. Rev Inst Med Trop Sao Paulo. 2007:49(5):279-87.

28. Salvador S, Ribeiro R, Winckler MI, Ohlweiler L, Riesgo R. Pediatric neurotoxocariasis with concomitant cerebral, cerebellar, and peripheral nervous system involvement: case report and review of the literature. J Pediatr (Rio J). 2010;86(6):531-4.

29. Pawlowski Z. Toxocariasis in humans: clinical expression and treatment dilemma. J Helminthol. 2001;75(4):299-305.

30. Taylor MR, Keane CT, O'Connor P, Girdwood RW, Smith H. Clinical features of covert toxocariasis. Scand J Infect Dis. 1987;19(6):693-6.

31. Glickman LT, Shofer FS. Zoonotic visceral and ocular larva migrans. Vet Clin North Am Small Anim Pract. 1987;17(1):39-53.

32. Mazur-Melewska K, Mania A, Figlerowicz M, Kemnitz P, Sluzewski W. Michalak M. The influence of age on a clinical presentation of Toxocara spp infection in children Ann Agric Environ Med. 2012;19(2):233-6.

33. Crum-Cianflone NF. Nonbacterial myositis. Curr Infect Dis Rep. 2010;12(5):374-82.

34. Glickman LT, Schantz PM. Epidemiology and pathogenesis of zoonotic toxocariasis. Epidemiol Rev. 1981;3:230-50.

35. Paul M, Stefaniak J, Twardosz-Pawlik H, Pecold K. The cooccurrence of Toxocara ocular and visceral larva migrans syndrome: a case series. Cases J. 2009;2:6881.

36. Taylor MR. The epidemiology of ocular toxocariasis. J Helminthol. 2001;75(2):109-18.
37. Stewart JM, Cubillan LD, Cunningham Jr ET. Prevalence, clinical features, and causes of vision loss among patients with ocular toxocariasis. Retina. 2005;25(8):1005-13.

38.• Fillaux J, Magnaval JF. Laboratory diagnosis of human toxocariasis. Vet Parasitol. 2013;193(4):327-36. Summary of the current laboratory diagnostic methods available for the diagnosis of human toxocariasis.

39. Vegh M, Danka J. The disease picture and treatment of Toxocara canis uveitis in childhood. lin Monbl Augenheilkd. 1987;191(5): 395-6.

40. Hotez PJ. Visceral and ocular larva migrans. Semin Neurol. 1993;13(2):175-9.

41. Abdel Razek AA, Watcharakorn A, Castillo M. Parasitic diseases of the central nervous system. Neuroimaging Clin N Am. 2011;21(4): 815-41. viii.

42. Quattrocchi G, Nicoletti A, Marin B, Bruno E, Druet-Cabanac M, Preux PM. Toxocariasis and epilepsy: systematic review and metaanalysis. PLoS Negl Trop Dis. 2012;6(8):e1775.

43. Walsh MG, Haseeb MA. Reduced cognitive function in children with toxocariasis in a nationally representative sample of the United States. Int J Parasitol. 2012;42(13-14):1159-63.

44. Gavignet B, Piarroux R, Aubin F, Millon L, Humbert P. Cutaneous manifestations of human toxocariasis. J Am Acad Dermatol. 2008;59(6):1031-42.

45. Cooper PJ. Interactions between helminth parasites and allergy. Curr Opin Allergy Clin Immunol. 2009;9(1):29-37.

46. Fitzsimmons CM, Dunne DW. Survival of the fittest: allergology or parasitology? Trends Parasitol. 2009;25(10):447-51.

47. Pinelli E, Brandes S, Dormans J, Gremmer E, van Loveren H. Infection with the roundworm Toxocara canis leads to exacerbation of experimental allergic airway inflammation. Clin Exp Allergy. 2008;38(4):649-58

48. Kanobana K, Vereecken K, Junco Diaz R, Sariego I, Rojas L, Bonet Gorbea $\mathrm{M}$, et al. Toxocara seropositivity, atopy and asthma: a study in Cuban schoolchildren. Trop Med Int Health. 2013;18(4):403-6.

49. Chen J, Zhou DH, Nisbet AJ, Xu MJ, Huang SY, Li MW, et al. Advances in molecular identification, taxonomy, genetic variation and diagnosis of Toxocara spp. Infect Genet Evol. 2012;12(7): 1344-8. Summary of the current state of knowledge and advances in molecular identification and diagnosis of Toxocara spp.

50. Kwon NH, Oh MJ, Lee SP, Lee BJ, Choi DC. The prevalence and diagnostic value of toxocariasis in unknown eosinophilia. Ann Hematol. 2006;85(4):233-8.

51. Glickman L, Schantz P, Dombroske R, Cypess R. Evaluation of serodiagnostic tests for visceral larva migrans. Am J Trop Med Hyg. 1978;27(3):492-8.

52. Mohamad S, Azmi NC, Noordin R. Development and evaluation of a sensitive and specific assay for diagnosis of human toxocariasis by use of three recombinant antigens (TES-26, TES-30USM, and TES-120). J Clin Microbiol. 2009;47(6):1712-7.

53. Magnaval JF, Faufingue JH, Morassin B, Fabre R. Eosinophil cationic protein, specific IgE and IgG4 in human toxocariasis. J Helminthol. 2006;80(4):417-23.

54. Watthanakulpanich D, Smith HV, Hobbs G, Whalley AJ, Billington D. Application of Toxocara canis excretory-secretory antigens and IgG subclass antibodies (IgG1-4) in serodiagnostic assays of human toxocariasis. Acta Trop. 2008;106(2):90-5.

55. Noordin R, Smith HV, Mohamad S, Maizels RM, Fong MY. Comparison of IgG-ELISA and IgG4-ELISA for Toxocara serodiagnosis. Acta Trop. 2005;93(1):57-62.

56. Gillespie SH, Bidwell D, Voller A, Robertson BD, Maizels RM. Diagnosis of human toxocariasis by antigen capture enzyme linked immunosorbent assay. J Clin Pathol. 1993;46(6):551-4.

57. Norhaida A, Suharni M, Liza Sharmini AT, Tuda J, Rahmah N. rTES-30USM: cloning via assembly PCR, expression, and 
evaluation of usefulness in the detection of toxocariasis. Ann Trop Med Parasitol. 2008;102(2):151-60.

58. Sharkey JA, McKay PS. Ocular toxocariasis in a patient with repeatedly negative ELISA titre to Toxocara canis. Br J Ophthalmol. 1993;77(4):253-4.

59. Schantz PM. Toxocara larva migrans now. Am J Trop Med Hyg. 1989;41(3 Suppl):21-34.

60. Felberg NT, Shields JA, Federman JL. Antibody to Toxocara canis in the aqueous humor. Arch Ophthalmol. 1981;99(9): $1563-4$.

61. Roldan W, Cornejo W, Espinoza Y. Evaluation of the dot enzymelinked immunosorbent assay in comparison with standard ELISA for the immunodiagnosis of human toxocariasis. Mem Inst Oswaldo Cruz. 2006;101(1):71-4.

62. de Visser L, Rothova A, de Boer JH, van Loon AM, Kerkhoff FT, Canninga-van Dijk MR, et al. Diagnosis of ocular toxocariasis by establishing intraocular antibody production. Am J Ophthalmol. 2008;145(2):369-74.

63. Lim JH. Toxocariasis of the liver: visceral larva migrans. Abdom Imaging. 2008;33(2):151-6.
64. Arevalo JF, Espinoza JV, Arevalo FA. Ocular toxocariasis. J Pediatr Ophthalmol Strabismus. 2013;50(2):76-86.

65. Sturchler D, Schubarth P, Gualzata M, Gottstein B, Oettli A. Thiabendazole vs. albendazole in treatment of toxocariasis: a clinical trial. Ann Trop Med Parasitol. 1989;83(5):473-8.

66. Barisani-Asenbauer T, Maca SM, Hauff W, Kaminski SL, Domanovits H, Theyer I, et al. Treatment of ocular toxocariasis with albendazole. J Ocul Pharmacol Ther. 2001;17(3):287-94.

67. Magnaval JF, Glickman LT, Dorchies P, Morassin B. Highlights of human toxocariasis. Korean J Parasitol. 2001;39(1):1-11.

68. Malafiej E, Spiewak E. The significance of the level of antibodies in the evaluation of the effects of treatment of toxocariasis. Wiad Parazytol. 2001;47(4):805-10.

69. Elefant GR, Shimizu SH, Sanchez MC, Jacob CM, Ferreira AW. A serological follow-up of toxocariasis patients after chemotherapy based on the detection of $\operatorname{IgG}$, IgA, and IgE antibodies by enzymelinked immunosorbent assay. J Clin Lab Anal. 2006;20(4):164-72.

70. Magnaval JF, Berry A, Fabre R, Morassin B. Eosinophil cationic protein as a possible marker of active human Toxocara infection. Allergy. 2001;56(11):1096-9. 